\title{
Wardrop Equilibrium for CDMA Systems
}

\author{
Nicolas Bonneau*, Mérouane Debbah ${ }^{\dagger}$, Eitan Altman*, and Are Hjørungnes ${ }^{\ddagger}$ \\ * MAESTRO, INRIA Sophia Antipolis, 2004 Route des Lucioles, B.P. 93, 06902 Sophia Antipolis, France \\ Email: \{nicolas.bonneau, eitan.altman\}@sophia.inria.fr \\ ${ }^{\dagger}$ Mobile Communications Group, Institut Eurecom, 2229 Route des Cretes, B.P. 193, 06904 Sophia Antipolis, France \\ Email: merouane.debbah@eurecom.fr \\ ${ }^{\ddagger}$ UniK-University Graduate Center, University of Oslo, Instituttveien 25, P. O. Box 70, N-2027 Kjeller, Norway \\ Email: arehj@unik.no
}

\begin{abstract}
In this contribution, the performance of an uplink CDMA system is analyzed in the context of frequency selective fading channels. Using game theoretic tools, a useful framework is provided in order to determine the optimal power allocation when users know only their own channel (while perfect channel state information is assumed at the base station). We consider the realistic case of frequency selective channels. This scenario illustrates the case of decentralized schemes and aims at reducing the downlink signaling overhead. Various receivers are considered, namely the Matched filter, the MMSE filter and the optimum filter. The goal of this paper is to derive simple expressions for the non-cooperative Nash equilibrium as the number of mobiles becomes large. To that end we combine two asymptotic methodologies. The first is asymptotic random matrix theory which allows us to obtain explicit expressions for the impact of all other mobiles on any given tagged mobile. The second is the theory of non-atomic games along with the Wardrop equilibrium concept which allows us to compute good approximations of the Nash equilibrium as the number of mobiles grow. ${ }^{1}$
\end{abstract}

\section{INTRODUCTION}

Power allocation (PA) is an important topic in the context of Code Division Multiple Access (CDMA) systems, in the uplink as well as in the downlink. In particular, in the uplink, users need to transmit with enough power to achieve their requested quality of service, and not more, in order to minimize the amount of interference caused to other users. Thus, an efficient PA mechanism allows to prevent an excessive consumption of the limited ressources of the users.

Usual PA mechanisms are based on a centralized procedure, with the base station receiving training sequences from the users and signaling back the optimal power allocation for each user, possibly according to some rule of precedence [1]. However, this involves a non negligible overhead and numerous non informational transmissions.

A way to avoid the constraints of a centralized procedure is to implement a decentralized one where each user takes a decision on the transmission power. This is, for example, the case in ad-hoc networks applications. In this context, a natural framework is game theory, which studies competition (as well as cooperation) between independant actors. Tools of game theory have already been frequently used as a

\footnotetext{
${ }^{1}$ This work was supported by the BIONETS project http://www . bionets.org/ and by the Research Council of Norway and the French Ministry of Foreign Affairs through the Aurora project entitled "Optimization of Broadband Wireless Communications Networks".
}

central framework for modeling competition and cooperation in networking, see for example [2] and references therein.

In the case of fading multiple-access channels, a game theoretic framework has already been proposed in [3]. Users compete with rates as utility and powers as moves in the game. However, their results rely on the fact that each user has a complete knowledge of the system, and in particular, perfect channel state information (CSI) of all users in the cell. This is a necessary requirement in order to use the theory of games of complete information, and a usual assumption in many papers in the field, as the authors point out. Nevertheless, it is rarely possible in practice and one can usually only satisfy at best the requirements knowing only its own channel.

When one knows only its own channel, other designs may be introduced. In [4], defining the utility as the ratio of the throughput to the transmission power, the authors obtain results of existence and unicity of a Nash equilibrium in this hypothesis. As far as the attenuation is concerned, only flat fading is considered in [4] and in [5], in the case of multiple carriers (each one being flat fading). However, wireless transmissions generally suffer from the effect of multiple paths, thus becoming frequency-selective. The goal of this paper is to determine the influence of the number of paths (or the selectivity of the channel) on the performance of PA. This work is an extension of [4] in the case of frequency-selective fading, in the framework of asymptotic CDMA with a cyclic prefix. We do not consider multiple carriers, as in [5], and the results are very different to those obtained in this work. The extension is not trivial and involves advanced results on random matrices with non-equal variances due to Girko [6] whereas classical results rely on the work of Silverstein [7].

In particular, we quantify the gain of the non-uniform PA with respect to uniform PA, according to the number of paths. The originality of the paper lies in the fact that we show that as the number of paths increases, the optimal PA becomes more and more uniform due to the ergodic behavior of all the CDMA channels. This is reminiscent of an effect ("channel hardening") already revealed in MIMO [8]. The highest gain (in terms of utility) is obtained in the case of flat fading (which also favors dis-uniform power allocation between the users).

In order to obtain analytical expressions, we consider the system in an asymptotic setting, letting both the number of users and the spreading factor tend to infinity with a fixed 
ratio. We use tools of random matrix theory [9] to analyze the system in this limit. Random matrix theory is a field of mathematical physics that has been recently applied to wireless communications to analyze various measures of interest such as capacity or Signal to Interference plus Noise Ratio (SINR). Interestingly, it enables to single out the main parameters of interest that determine the performance in numerous models of communication systems [10], [11]. In addition, these asymptotic results provide good approximations for the practical finite size case, as shown by simulations.

In the asymptotic regime, the non-cooperative game becomes a non-atomic one, in which the impact (through interference) of any single mobile on the performance of other mobiles is negligible. In the networking game context, the related solution concept is often called Wardrop equilibrium [12]. The primary application of Wardrop equilibrium concerns road traffic and flows over a network. It is often much easier to compute than the original Nash equilibrium [2], and yet, the former equilibrium is a good approximation for the latter, see details in [13].

The layout of this paper is the following. First we introduce useful notations and concepts of random matrix theory in Sec. II, and our communication model in Sec. III. Asymptotic SINR and capacity expressions are given in Sec. IV. The game between users is introduced and the Nash equilibrium is derived in Sec. V. Finally, theoretical results for the power allocation are derived in Sec. VI and are matched with simulations in Sec. VII.

\section{NOTATIONS}

The following definitions and theorem can be found in [9] and will be used in the following sections. In this section, $N$ and $K$ are positive integers.

Definition 1: Let $\nu$ be a probability measure. The Stieltjes transform $m^{\nu}$ associated to $\nu$ is given by

$$
m^{\nu}(z)=\int \frac{1}{t-z} \nu(d t)
$$

Definition 2: Let $\mathbf{v}=\left[v_{1}, \ldots, v_{N}\right]$ be a vector. Its empirical distribution is the function $F_{N}^{\mathbf{v}}: \mathbb{R} \rightarrow[0,1]$ defined by:

$$
F_{N}^{\mathbf{v}}(x)=\frac{1}{N} \#\left\{v_{i} \leq x \mid i=1 \ldots N\right\} .
$$

In other words, $F_{N}^{\mathbf{v}}(x)$ is the fraction of elements of $\mathbf{v}$ that are inferior or equal to $x$. In particular, if $\mathbf{v}$ is the vector of eigenvalues of a matrix $\mathbf{V}, F_{N}^{\mathbf{v}}$ is called the empirical eigenvalue distribution of $\mathbf{V}$.

Definition 3: Let $\mathbf{V}$ be a $N \times K$ random matrix with independant columns and entries $v_{i j}$. Denote by $\lfloor\cdot\rfloor$ the closest smaller integer. $\mathbf{V}$ is said to behave ergodically if, as $N, K \rightarrow$ $\infty$ with $K / N \rightarrow \alpha$, for $x \in[0,1]$, the empirical distribution of

$$
\left[\left|v_{\lfloor x N\rfloor, 1}\right|^{2}, \ldots,\left|v_{\lfloor x N\rfloor, K}\right|^{2}\right]
$$

converges almost surely to a non-random limit distribution denoted $F_{x}^{\mathbf{V}}(\cdot)$ and, for $y \in[0, \alpha]$, the empirical distribution of

$$
\left[\left|v_{1,\lfloor y N\rfloor}\right|^{2}, \ldots,\left|v_{N,\lfloor y N\rfloor}\right|^{2}\right]
$$

converges almost surely to a non-random limit distribution denoted $F_{y}^{\mathbf{V}}(\cdot)$.

Definition 4: Let $\mathbf{V}$ be a $N \times K$ random matrix that behaves ergodically as in Def. 3, such as $F_{x}^{\mathbf{V}}(\cdot)$ and $F_{y}^{\mathbf{V}}(\cdot)$ have all their moments bounded. The two-dimensional channel profile of $\mathbf{V}$ is the function $\rho^{\mathbf{V}}(x, y):[0,1] \times[0, \alpha] \rightarrow \mathbb{R}$ such that, if the random variable $X$ is uniformly distributed in $[0,1]$, then the distribution of $\rho^{\mathbf{V}}(X, y)$ equals $F_{y}^{\mathbf{V}}(\cdot)$ and, if the random variable $Y$ is uniformly distributed in $[0, \alpha]$, then the distribution of $\rho^{\mathbf{V}}(x, Y)$ equals $F_{x}^{\mathbf{V}}(\cdot)$.

Theorem 1: Let $\mathbf{Y}=\mathbf{V} \odot \mathbf{W}$ be a $N \times K$ matrix, where $\odot$ is the Hadamard (element-wise) product and $\mathbf{V}$ and $\mathbf{W}$ are independent $N \times K$ random matrices. Assume that $\mathbf{V}$ behaves ergodically with channel profile $\rho^{\mathbf{V}}(x, y)$ as in Def. 4 and that $\mathbf{W}$ has i.i.d. entries with zero mean and variance $\frac{1}{N}$. Then, as $N, K \rightarrow \infty$ with $K / N \rightarrow \alpha$, the empirical eigenvalue distribution of $\mathbf{Y} \mathbf{Y}^{H}$ converges almost surely to a non-random limit distribution function whose Stieltjes transform is given by:

$$
\begin{aligned}
m^{\mathbf{Y Y}} \mathbf{Y}^{H}(z) & =\lim _{N \rightarrow \infty} \frac{1}{N} \operatorname{Trace}\left(\left(\mathbf{Y} \mathbf{Y}^{H}-z \mathbf{I}\right)^{-1}\right) \\
& =\int_{0}^{1} u(x, z) d x
\end{aligned}
$$

and $u(x, z)$ satisfies the fixed point equation:

$$
u(x, z)=\frac{1}{\int_{0}^{\alpha} \frac{\rho^{\mathbf{v}}(x, y) d y}{1+\int_{0}^{1} \rho^{\mathbf{v}}\left(x^{\prime}, y\right) u\left(x^{\prime}, z\right) d x^{\prime}}-z} .
$$

The solution to equation (1) exists and is unique in the class of functions $u(x, z) \geq 0$, analytic for $\operatorname{Im}(z)>0$, and continuous on $x \in[0,1]$.

\section{MODEL}

We consider a single CDMA cell, i.e., inter-cell interference free case. The spreading length is denoted $N$. The number of users in the cell is $K$. The load is $\alpha=K / N$. The general case of wide-band CDMA is considered where the signal transmitted by user $k$ has complex envelope

$$
x_{k}(t)=\sum_{n} s_{k n} v_{k}(t-n T) .
$$

$v_{k}(t)$ is an weighted sum of elementary modulation pulses which satisfy the Nyquist criterion with respect to the chip interval $T_{c}\left(T=N T_{c}\right)$ :

$$
v_{k}(t)=\sum_{\ell=1}^{N} v_{\ell k} \psi\left(t-(\ell-1) T_{c}\right) .
$$

The signal is transmitted over a frequency selective channel with impulse response $c_{k}(\tau)$. Under the assumption of slowlyvarying fading, the continuous time received signal $y(t)$ at the base station has the form:

$$
y(t)=\sum_{n} \sum_{k=1}^{K} s_{k n} \int c_{k}(\tau) v_{k}(t-n T-\tau) d \tau+n(t)
$$


where $n(t)$ is zero-mean complex white Gaussian noise with variance $\sigma^{2}$. The signal (after pulse matched filtering by $\psi^{*}(-t)$ ) is sampled at the chip rate to get a discrete-time signal that has the form:

$$
\mathbf{y}=\sum_{k=1}^{K} \mathbf{C}_{k} \mathbf{v}_{k} \sqrt{P_{k}} s_{k}+\mathbf{n}
$$

where $\mathbf{C}_{k}$ are $N \times N$ Toeplitz matrices representing the frequency selective fading for the $k$-th user and $\mathbf{n}$ is an $N \times 1$ Additive White Gaussian Noise (AWGN) vector with covariance matrix $\sigma^{2} \mathbf{I}_{N}$.

Since the users are supposed to be synchronized with the base station and for sake of simplicity, we will consider in all the following that users add a cyclic prefix of length equal to the channel impulse response length to their code sequence. ${ }^{2}$ This case is similar to uplink MC-CDMA [15], [16] and as a consequence, $\left\{\mathbf{C}_{k}\right\}$ becomes circulant [17] and can be diagonalized in a Fourier basis F. Model (2) simplifies therefore to:

$$
\mathbf{y}=\sum_{k=1}^{K} \mathbf{F H}_{k} \mathbf{F}^{H} \mathbf{v}_{k} \sqrt{P_{k}} s_{k}+\mathbf{n}
$$

where $\mathbf{H}_{k}$ is a diagonal matrix with diagonal elements $\left\{h_{i k}\right\}_{i=1 \ldots N}$. For each user $k$, the coefficients $h_{i k}$ are the discrete Fourier transform of the channel impulse response. We make the hypothesis that the users employ Gaussian i.i.d. codes [18]. Since every unitary tranformation of a Gaussian i.i.d. vector is a Gaussian i.i.d. vector (so that $\mathbf{w}_{i}=\mathbf{F}^{H} \mathbf{v}_{i}$ has the same distribution as $\mathbf{v}_{i}$ for any $i$ ), one can multiply $\mathbf{y}$ in (3) with $\mathbf{F}^{H}$ and obtain without any change in the statistics:

$$
\begin{aligned}
\mathbf{y} & =\sum_{k=1}^{K} \mathbf{H}_{k} \mathbf{w}_{k} \sqrt{P_{k}} s_{k}+\mathbf{n} \\
& =(\mathbf{H} \sqrt{\mathbf{P}} \odot \mathbf{W}) \mathbf{s}+\mathbf{n}
\end{aligned}
$$

where $\odot$ is the Hadamard product.

In (4), $\mathbf{H}$ is the frequency selective fading matrix, of size $N \times K$ :

$$
\mathbf{H}=\left[\begin{array}{cccc}
h_{11} & h_{12} & \ldots & h_{1 K} \\
\vdots & \vdots & & \vdots \\
h_{N 1} & h_{N 2} & \ldots & h_{N K}
\end{array}\right] .
$$

$\sqrt{\mathbf{P}}$ is the root square of the diagonal power control matrix, of size $K \times K$.

W is an $N \times K$ random spreading matrix:

$$
\mathbf{W}=\left[\mathbf{w}_{1}\left|\mathbf{w}_{2}\right| \cdots \mid \mathbf{w}_{K}\right] \text { where } \mathbf{w}_{k}=\left[\begin{array}{c}
w_{1 k} \\
\vdots \\
w_{N k}
\end{array}\right] .
$$

Note that asymptotically (as $N \rightarrow \infty$ ), for a given multipath channel of length $L$, model (4) is also valid for the case of uplink DS-CDMA since all Toeplitz matrices can be asymptotically diagonalized in a Fourier Basis [14], [19].

\footnotetext{
${ }^{2}$ Note that in the asymptotic case (when $N \rightarrow \infty$ ), the result holds without the need of a cyclic prefix as long as the channel is absolutely summable [14].
}

In the following, we will assume that the frequency selective fading matrix $\mathbf{H}$ behaves ergodically, as in Def. 3. The twodimensional channel profile of $\mathbf{H} \sqrt{\mathbf{P}}$ is denoted $\rho(f, x)=$ $P(x)|h(f, x)|^{2}, f \in[0,1], x \in[0, \alpha] . f$ is the frequency index and $x$ is the user index. This enables us to use Th. 1 in order to obtain expressions for the SINR.

\section{ASYMPTOTIC SINR EXPRESSIONS}

Let $\mathbf{h}_{k}$ be the $k$-th column of $\mathbf{H}$, and $\mathbf{H}_{(-k)}$ be $\mathbf{H}$ with $\mathbf{h}_{k}$ removed. Similarly, let $\mathbf{w}_{k}$ be the $k$-th column of $\mathbf{W}$, and $\mathbf{W}_{(-k)}$ be $\mathbf{W}$ with $\mathbf{w}_{k}$ removed. Let $\sqrt{\mathbf{P}}(-k)$ be $\sqrt{\mathbf{P}}$ with the $k$-th column and line removed. Finally, let $\mathbf{G}_{(-k)}=$ $\mathbf{H}_{(-k)} \sqrt{\mathbf{P}}_{(-k)} \odot \mathbf{W}_{(-k)}$.

\section{A. Matched Filter}

Supposing perfect CSI at the receiver, the matched filter for the $k$-th user is given by $\mathbf{g}_{k}=\left(\mathbf{h}_{k} \sqrt{P_{k}} \odot \mathbf{w}_{k}\right)$. This leads to the following expression for the SINR of user $k$

$$
\operatorname{SINR}_{k}=\frac{\left|\mathbf{g}_{k}^{H} \mathbf{g}_{k}\right|^{2}}{\sigma^{2} \mathbf{g}_{k}^{H} \mathbf{g}_{k}+\mathbf{g}_{k}^{H}\left(\mathbf{G}_{(-k)} \mathbf{G}_{(-k)}^{H}\right) \mathbf{g}_{k}} .
$$

Proposition 1: [11] As $N, K \rightarrow \infty$ with $K / N \rightarrow \alpha$, the SINR of user $k$ at the output of the matched filter is given by

$$
\operatorname{SINR}_{k}=\beta^{\mathrm{MF}}\left(\frac{k}{N}\right)
$$

where $\beta^{\mathrm{MF}}:[0, \alpha] \rightarrow \mathbb{R}$ is given by

$$
\begin{aligned}
& \beta^{\mathrm{MF}}(x)=P(x) . \\
& \frac{\left(\int_{0}^{1}|h(f, x)|^{2} d f\right)^{2}}{\sigma^{2} \int_{0}^{1}|h(f, x)|^{2} d f+\int_{0}^{\alpha} \int_{0}^{1} P(y)|h(f, y)|^{2}|h(f, x)|^{2} d f d y} .
\end{aligned}
$$

Denoting $\operatorname{SINR}_{k}=\beta_{k}$, we observe that $P_{k} \frac{\partial \beta_{k}}{\partial P_{k}}=\beta_{k}$.

\section{B. MMSE Filter}

Supposing perfect CSI at the receiver, the MMSE filter for the $k$-th user is given by $\mathbf{g}_{k}^{\mathrm{MMSE}}=\mathbf{R}^{-1} \mathbf{g}_{k}$, where $\mathbf{R}=$ $\left((\mathbf{H} \sqrt{\mathbf{P}} \odot \mathbf{W})(\mathbf{H} \sqrt{\mathbf{P}} \odot \mathbf{W})^{H}+\sigma^{2} \mathbf{I}_{N}\right)$. This leads to the following expression for the SINR of user $k$

$$
\operatorname{SINR}_{k}=\mathbf{g}_{k}^{H}\left(\mathbf{G}_{(-k)} \mathbf{G}_{(-k)}^{H}+\sigma^{2} \mathbf{I}_{N}\right)^{-1} \mathbf{g}_{k} .
$$

Proposition 2: [11] As $N, K \rightarrow \infty$ with $K / N \rightarrow \alpha$, the SINR of user $k$ at the output of the MMSE receiver is given by:

$$
\operatorname{SINR}_{k}=\beta\left(\frac{k}{N}\right)
$$

where $\beta:[0, \alpha] \rightarrow \mathbb{R}$ is a function defined by the implicit equation

$$
\beta(x)=P(x) \int_{0}^{1} \frac{|h(f, x)|^{2} d f}{\sigma^{2}+\int_{0}^{\alpha} \frac{P(y)|h(f, y)|^{2} d y}{1+\beta(y)}} .
$$

Denoting $\mathrm{SINR}_{k}=\beta_{k}$, we observe that $P_{k} \frac{\partial \beta_{k}}{\partial P_{k}}=\beta_{k}$. 
From Prop. 2, we have the capacity of user $k$

$$
C_{k}^{\mathrm{MMSE}}=\frac{1}{N} \log _{2}\left(1+\beta_{k}\right) .
$$

The global capacity of the system is

$$
C^{\mathrm{MMSE}}=\int_{0}^{\alpha} \log _{2}(1+\beta(x)) d x .
$$

\section{Optimal Filter}

The term optimal filter designates a filter capable of decoding the received signal at the bound given by Shannon's capacity. Hence it is difficult to define an SINR associated to it. However, results of random matrix theory can still be applied. Let $\mathbf{Y}=(\mathbf{H} \sqrt{\mathbf{P}} \odot \mathbf{W})$. The definition of Shannon's capacity per dimension for our system is

$$
C_{(N)}^{\mathrm{OPT}}=\frac{1}{N} \log _{2} \operatorname{det}\left(\mathbf{I}_{N}+\frac{1}{\sigma^{2}} \mathbf{Y} \mathbf{Y}^{H}\right) .
$$

As $N, K \rightarrow \infty$ with $K / N \rightarrow \alpha$,

$$
C_{(N)}^{\mathrm{OPT}} \rightarrow \int \log _{2}\left(1+\frac{1}{\sigma^{2}} t\right) \nu(d t)
$$

where $\nu$ is the empirical eigenvalue distribution of $\mathbf{Y} \mathbf{Y}^{H}$, as in Def. 2. If we differentiate the asymptotic value $C^{\mathrm{OPT}}$ of (9) with respect to $\sigma^{2}$, we obtain

$$
\begin{aligned}
\frac{\partial C^{\mathrm{OPT}}}{\partial \sigma^{2}} & =\log _{2}(e) \int \frac{-\frac{1}{\sigma^{4}} t}{1+\frac{1}{\sigma^{2}} t} \nu(d t) \\
& =\log _{2}(e) \int \frac{\sigma^{2}\left(-\frac{1}{\sigma^{4}} t-\frac{1}{\sigma^{2}}+\frac{1}{\sigma^{2}}\right)}{\sigma^{2}\left(1+\frac{1}{\sigma^{2}} t\right)} \nu(d t) \\
& =\log _{2}(e)\left(\int \frac{1}{t+\sigma^{2}} \nu(d t)-\frac{1}{\sigma^{2}} \int \nu(d t)\right) \\
& =\log _{2}(e)\left(m^{\nu}\left(-\sigma^{2}\right)-\frac{1}{\sigma^{2}}\right)
\end{aligned}
$$

where $m^{\nu}(\cdot)$ is the the Stieltjes transform of the empirical eigenvalue distribution of $\mathbf{Y} \mathbf{Y}^{H}$. From Th. 1, $m^{\nu}(\cdot)$ is given by

$$
m^{\nu}(z)=\int_{0}^{1} u(f, z) d f
$$

where $u(f, z)$ is given by (1) with $\rho^{\mathbf{H} \sqrt{\mathbf{P}}}(f, x)=\rho(f, x)=$ $P(x)|h(f, x)|^{2}$. Given that if $\sigma^{2}=+\infty, C^{\mathrm{OPT}}=0$, it is immediate to obtain $C^{\mathrm{OPT}}$ from (10) as

$$
C^{\mathrm{OPT}}=\log _{2}(e) \int_{\sigma^{2}}^{+\infty} m^{\nu}(-z)-\frac{1}{z} d z .
$$

Proposition 3: $C^{\mathrm{OPT}}$ and $C^{\mathrm{MMSE}}$ are related through the following equality

$$
\begin{aligned}
C^{\mathrm{OPT}} & =C^{\mathrm{MMSE}}-\log _{2}(e) \int_{0}^{\alpha} \frac{\beta(x)}{1+\beta(x)} d x \\
& +\int_{0}^{1} \log _{2}\left(1+\frac{1}{\sigma^{2}} \int_{0}^{\alpha} \frac{\rho(f, x)}{1+\beta(x)} d x\right) d f .
\end{aligned}
$$

Proof: See Appendix.
Assuming perfect cancellation of decoded users, successive interference cancellation with MMSE filter achieves the optimum capacity [20]. The following proposition ensues from this fact.

Proposition 4: [11] As $N, K \rightarrow \infty$ with $K / N \rightarrow \alpha$, the optimal capacity is given by:

$$
C^{\mathrm{OPT}}=\int_{0}^{\alpha} \log _{2}\left(1+\beta^{\mathrm{SIC}}(x)\right) d x
$$

where $\beta^{\text {SIC }}:[0, \alpha] \rightarrow \mathbb{R}$ is a function defined by the implicit equation

$$
\beta^{\mathrm{SIC}}(x)=P(x) \int_{0}^{1} \frac{|h(f, x)|^{2} d f}{\sigma^{2}+\int_{0}^{x} \frac{P(y)|h(f, y)|^{2} d y}{1+\beta^{\operatorname{SIC}}(y)}} .
$$

Prop. 4 enables us to extract an expression that is analog to the SINR for the optimal filter. This expression obeys the property $P_{k} \frac{\partial \beta_{k}}{\partial P_{k}}=\beta_{k}$.

\section{UTILITY AND NASH EQUILIBRIUM}

From now on, we denote $\operatorname{SINR}_{k}=\beta_{k}$, whichever filter is actually used. In order to place ourselves in a game theoretic setting, we have to define a utility for the users. Utility measures the gain of a user as a result of the strategy this user plays. The strategy for a mobile is its power allocation. As in [4], it is natural to define utility as the ratio of some measure of performance $\gamma$ and the transmit power. For example, in the simulations, we consider the goodput $\gamma\left(\beta_{k}\right)=\left(1-e^{-\beta_{k}}\right)^{M}$ [21], [4] where $M$ is the number of bits transmitted in a CDMA packet (e.g., $M=100$ in the simulations). This is a relevant performance measure, as each mobile wants to use its (limited) battery power to ensure that the maximum possible amount of information is correctly decoded at the receiver. Therefore, the utility of user $k$ can be written

$$
u_{k}=\frac{\gamma_{k}}{P_{k}} \text {. }
$$

This utility is expressed in bits per joule.

In the non-cooperative game setting, all users transmit concurrently. The purpose of each user is to selfishly maximize its utility. A Nash equilibrium is obtained when no user can benefit by unilaterally deviating from its strategy.

We denote $\gamma_{k}=\gamma\left(\beta_{k}\right)$, where $\gamma(\cdot)$ is the same function for all users. To obtain the maximum utility achievable by user $k$, we differentiate $u_{k}$ with respect to the power $P_{k}$ and equate to 0 . We obtain

$$
P_{k} \frac{\partial \beta_{k}}{\partial P_{k}} \gamma^{\prime}\left(\beta_{k}\right)-\gamma\left(\beta_{k}\right)=0 .
$$

For all filters under consideration, (5), (6) and (13) imply $P_{k} \frac{\partial \beta_{k}}{\partial P_{k}}=\beta_{k}$, thus (15) reduces an equation on $\beta_{k}$

$$
\beta_{k} \gamma^{\prime}\left(\beta_{k}\right)-\gamma\left(\beta_{k}\right)=0
$$

Eq. (16) is particularly interesting in the case when there exists a unique solution $\beta^{\star}$. The existence of a solution to (16) is guaranteed as long as the function $\gamma(\cdot)$ is a quasiconcave function of the SINR, i.e., there exists a point below which the function is non-decreasing, and above which the function is 
non-increasing [21]. In addition, we assume that the function $\gamma(\cdot)$ takes value $\gamma(0)=0$, so that users cannot achieve an infinite utility by not transmitting. This occurs for several functions $\gamma(\cdot)$ of interest, in particular the goodput [21], [4], which we will use for simulations.

It is not interesting to consider the capacity as a function $\gamma(\cdot)$ in the utility. It would lead to the trivial result $\beta^{\star}=0$, meaning that the Nash equilibrium is achieved when mobiles never transmit.

The uniqueness of the solution $\beta^{\star}$ to (16) is due to fact that the SINR of each user is a strictly increasing function of its transmit power. Given the target SINR $\beta^{\star}$, we obtain the strategy of users in the next section.

\section{Power Allocation in the Nash Equilibrium}

\section{A. Flat Fading}

In this subsection, we show that the results of [4] for Matched and MMSE filters are a special case of our setting when $L=1$ (flat fading case). In addition, we derive the power allocation for the Optimum filter. When there is only one path, for each user $k$, denoted by its index $\frac{k}{N}=x \in[0, \alpha], h(f, x)$ does not depend on $f$. Given the target SINR $\beta^{\star}$, we have explicit expressions of the power with which user $k$ transmits for the various receivers.

The Wardrop equilibrium arises naturally when the game is considered to be played by infinitely many users, so that each user has a negligible effect on the global equilibrium. Wardrop's principle states that The journey times in all routes actually used are equal and less than those which would be experienced by a single vehicle on any unused route. In our setting, we interpret this principle in the following way: The power allocation of one user does not depend on the power allocation of the others. We thus show that in the asymptotic limit, we obtain that the strategy used by each user does not influence the strategy of other users.

1) Matched filter: From Prop. 1, the continuous formulation is

$$
P(x)=\frac{\beta^{\star}\left(\sigma^{2}+\int_{0}^{\alpha} P(y)|h(y)|^{2} d y\right)}{|h(x)|^{2}}
$$

or equivalently in a discrete form

$$
P_{k}=\frac{\beta^{\star}\left(\sigma^{2}+\frac{1}{N} \sum_{j=1, j \neq k}^{K} P_{j}\left|h_{j}\right|^{2}\right)}{\left|h_{k}\right|^{2}} .
$$

Summing (17) over $k=1, \ldots, K$, we obtain a closed form expression for the minimum power with which user $k$ transmits when using the matched filter

$$
P_{k}=\frac{1}{\left|h_{k}\right|^{2}} \frac{\sigma^{2} \beta^{\star}}{1-\alpha \beta^{\star}} \text { for } \alpha<\frac{1}{\beta^{\star}} .
$$

is

2) MMSE filter: From Prop. 2, the continuous formulation

$$
P(x)=\frac{\beta^{\star}\left(\sigma^{2}+\frac{1}{1+\beta^{\star}} \int_{0}^{\alpha} P(y)|h(y)|^{2} d y\right)}{|h(x)|^{2}} .
$$

or equivalently in a discrete form

$$
P_{k}=\frac{\beta^{\star}\left(\sigma^{2}+\frac{1}{1+\beta^{\star}} \frac{1}{N} \sum_{j=1, j \neq k}^{K} P_{j}\left|h_{j}\right|^{2}\right)}{\left|h_{k}\right|^{2}} .
$$

Summing (19) over $k=1, \ldots, K$, we obtain a closed form expression for the minimum power with which user $k$ transmits when using the MMSE filter

$$
P_{k}=\frac{1}{\left|h_{k}\right|^{2}} \frac{\sigma^{2} \beta^{\star}}{1-\alpha \frac{\beta^{\star}}{1+\beta^{\star}}} \text { for } \alpha<1+\frac{1}{\beta^{\star}} .
$$

Both (18) and (20) are the same results as in [4].

3) Optimum filter: In the case of the optimum filter, the SINR is not defined. However, the target capacity is the same as for the other receivers, i.e., $C^{\star}=\frac{1}{N} \log _{2}\left(1+\beta^{\star}\right)$.

Proposition 5: The power allocation is given by

$$
P_{k}=\frac{1}{\left|h_{k}\right|^{2}} \frac{\sigma^{2} \beta^{+}}{1-\alpha \frac{\beta^{+}}{1+\beta^{+}}} \text {for } \alpha<1+\frac{1}{\beta^{+}}
$$

where $\beta^{+}$is the solution to

$$
\begin{aligned}
& \alpha \log _{2}\left(1+\beta^{+}\right)-\alpha \log _{2}(e) \frac{\beta^{+}}{1+\beta^{+}} \\
& \quad+\log _{2}\left(1+\frac{1}{1+\beta^{+}} \frac{\alpha \beta^{+}}{1-\alpha \frac{\beta^{+}}{1+\beta^{+}}}\right)=\alpha \log _{2}\left(1+\beta^{\star}\right) .
\end{aligned}
$$

Proof: See Appendix.

\section{B. Frequency Selective Fading}

In the context of frequency selective fading, for each user $k$, denoted by its index $\frac{k}{N}=x \in[0, \alpha]$, there are $L>1$ paths with respective attenuations $h_{\ell}(x), \ell=1, \ldots, L$, which are i.i.d. random variables with some known distribution. We suppose that $h_{\ell}(x)$ has mean zero, and the distributions of the real part and imaginary part of $h_{\ell}(x)$ are even functions, as for example the Gaussian distribution, which we consider in the simulations. $h(f, x)$ depends on $f$ through $h(f, x)=\sum_{\ell=1}^{L} h_{\ell}(x) e^{-2 \pi i f(\ell-1)}$. Given the target SINR $\beta^{\star}$, the Nash equilibrium power allocation is determined by implicit equations for the various receivers.

1) Matched filter: The continuous formulation is

$$
\begin{aligned}
& P(x)=\beta^{\star} . \\
& \frac{\sigma^{2} \int_{0}^{1}|h(f, x)|^{2} d f+\int_{0}^{1} \int_{0}^{\alpha} P(y)|h(f, y)|^{2}|h(f, x)|^{2} d f d y}{\left(\int_{0}^{1}|h(f, x)|^{2} d f\right)^{2}}
\end{aligned}
$$

or equivalently in a discrete form

$$
\begin{aligned}
& P_{k}=\beta^{\star} . \\
& \frac{\frac{\sigma^{2}}{N} \sum_{n=1}^{N}\left|h_{n k}\right|^{2}+\frac{1}{N} \sum_{n=1}^{N}\left|h_{n k}\right|^{2} \frac{1}{N} \sum_{j=1, j \neq k}^{K} P_{j}\left|h_{n j}\right|^{2}}{\left(\frac{1}{N} \sum_{n=1}^{N}\left|h_{n k}\right|^{2}\right)^{2}} .
\end{aligned}
$$

In (23), $h_{n k}=h\left(\frac{n-1}{N}, \frac{k}{N}\right)$. 
The problem with this expression is that the power allocation of user $k$ seems to depend on the power allocation and fading realization of all the other users. In order to alleviate this dependancy, we suppose that the number of users tends to infinity. Supposing that $\frac{1}{N} \sum_{j=1}^{K} P_{j}\left|h_{n j}\right|^{2}$ is asymptotically a constant, denoted $X$, we obtain

$$
X=\frac{\alpha \beta^{\star} \sigma^{2} \frac{1}{K} \sum_{j=1}^{K} \frac{\left|h_{n j}\right|^{2}}{E_{j}}}{1-\alpha \beta^{\star} \frac{1}{K} \sum_{j=1}^{K} \frac{\left|h_{n j}\right|^{2}}{E_{j}}}
$$

where $E_{j}=\frac{1}{N} \sum_{m=1}^{N}\left|h_{m j}\right|^{2}$. As $K \rightarrow \infty$, we can apply the Central Limit Theorem to the sum of random variables

$$
\frac{1}{K} \sum_{j=1}^{K} \frac{\left|h_{n j}\right|^{2}}{E_{j}} .
$$

It tends to its expectation, which is equal to 1 (see Appendix).

It follows that asymptotically $X=\frac{\alpha \beta^{\star} \sigma^{2}}{1-\alpha \beta^{\star}}$ (and simulations in Sec. VII prove that this approximation is valid for moderate finite values of $N$ ). From (23), we obtain a formula similar to (18)

$$
P_{k}=\frac{1}{E_{k}} \frac{\sigma^{2} \beta^{\star}}{1-\alpha \beta^{\star}} \text { for } \alpha<\frac{1}{\beta^{\star}} .
$$

2) MMSE filter: The continuous formulation is

$$
P(x)=\frac{\beta^{\star}}{\int_{0}^{1} \frac{|h(f, x)|^{2} d f}{\sigma^{2}+\frac{1}{1+\beta^{\star}} \int_{0}^{\alpha} P(y)|h(f, y)|^{2} d y}}
$$

or equivalently in a discrete form

$$
P_{k}=\frac{\beta^{\star}}{\frac{1}{N} \sum_{n=1}^{N} \frac{\left|h_{n k}\right|^{2}}{\sigma^{2}+\frac{1}{1+\beta^{\star}} \frac{1}{N} \sum_{j=1, j \neq k}^{K} P_{j}\left|h_{n j}\right|^{2}}} .
$$

In (28), $h_{n k}=h\left(\frac{n-1}{N}, \frac{k}{N}\right)$.

As previously, in order to alleviate the dependancy of the power of user $k$ on the power allocation of the other users, we suppose that the number of users tends to infinity. Supposing that $\frac{1}{N} \sum_{j=1}^{K} P_{j}\left|h_{n j}\right|^{2}$ is asymptotically a constant, denoted $X$, we obtain the following equation.

$$
X=\frac{\alpha \beta^{\star} \sigma^{2} \frac{1}{K} \sum_{j=1}^{K} \frac{\left|h_{n j}\right|^{2}}{E_{j}}}{1-\frac{\alpha \beta^{\star}}{1+\beta^{\star}} \frac{1}{K} \sum_{j=1}^{K} \frac{\left|h_{n j}\right|^{2}}{E_{j}}}
$$

where $E_{j}=\frac{1}{N} \sum_{m=1}^{N}\left|h_{m j}\right|^{2}$.

It follows that asymptotically $X=\frac{\alpha \beta^{\star} \sigma^{2}}{1-\alpha \frac{\beta^{\star}}{1+\beta^{\star}}}$, we obtain a formula similar to (20)

$$
P_{k}=\frac{1}{E_{k}} \frac{\sigma^{2} \beta^{\star}}{1-\alpha \frac{\beta^{\star}}{1+\beta^{\star}}} \text { for } \alpha<1+\frac{1}{\beta^{\star}} .
$$

3) Optimum filter: In the case of the optimum filter, the SINR is not defined. However, for a given $\beta^{\star}$, the capacity is the same as for the other receivers, i.e., $C^{\star}=\alpha \log _{2}\left(1+\beta^{\star}\right)$.

Proposition 6: Asymptotically, as $N, K \rightarrow \infty$, the power allocation is given by

$$
P_{k}=\frac{1}{E_{k}} \frac{\sigma^{2} \beta^{+}}{1-\alpha \frac{\beta^{+}}{1+\beta^{+}}} \text {for } \alpha<1+\frac{1}{\beta^{+}}
$$

where $\beta^{+}$is the solution to

$$
\begin{aligned}
& \alpha \log _{2}\left(1+\beta^{+}\right)-\alpha \log _{2}(e) \frac{\beta^{+}}{1+\beta^{+}} \\
& \quad+\log _{2}\left(1+\frac{1}{1+\beta^{+}} \frac{\alpha \beta^{+}}{1-\alpha \frac{\beta^{+}}{1+\beta^{+}}}\right)=\alpha \log _{2}\left(1+\beta^{\star}\right) .
\end{aligned}
$$

Proof: The proof is similar to the proof of Prop. 5. We observe that for all filters considered, the optimal PA is a constant times the inverse of the total energy of the channel $E_{j}$. Via Parseval's Theorem, $E_{j}=\sum_{\ell=1}^{L}\left|h_{\ell}\left(\frac{j}{N}\right)\right|^{2}$. It is a sum of i.i.d. random variables. As the number of paths increases, the optimal PA tends to a uniform PA. This is an effect similar to "channel hardening" [8]: as the number of paths increases, the variance of the distribution of the channel energy decreases and the Nash equilibrium PA becomes more and more uniform for all users.

\section{NuMERICAL RESULTS}

We consider a CDMA system with $K=32$ users and a spreading factor $N=256$. The noise variance is $\sigma^{2}=10^{-10}$. For a number of bits in a CDMA packet $M=100$, the goodput is $\gamma(\beta)=\left(1-e^{-\beta}\right)^{100}$ (see [4]), and $\beta^{\star}=6.48$. The capacity achieved at the Nash Equilibrium is $C=$ $\alpha \log _{2}\left(1+\beta^{\star}\right)=0.39$ bits/s. Unfortunately, the capacity itself cannot be used as a relevant performance measure in the definition of the utility, because in this case the maximal utility is obtained when not sending.

We have performed simulations over 10000 realizations. Fig. 1 shows the good fit of theoretic values calculated directly from (26), (30) and (31) with those simulations. We see that optimum filter requires the minimal power, and matched filter the maximal power to achieve the required BER.

In Fig. 2 we have plotted the average utility versus the number of multipaths $L$. Multipaths are supposed to be i.i.d. Rayleigh distributed with variance $1 / L$, in order for the channels to have the same energy. Two cases are considered: the utility obtained in the Nash equilibrium, according to the PA given by (23) and (28), and the utility in the case where all nodes transmit at the same power. In order to compare, the sum of the uniform powers is equal to the sum of the powers used in the Nash equilibrium. The utility does not vary with $L$ in the Nash equilibrium: the Central Limit Theorem applies to the utility, which is a constant times the random variable $E_{k}$ in the Nash equilibrium. The utility with uniform powers is always inferior to the utility in the Nash equilibrium. However, as $L$ increases, the gap decreases, as the variance of $E_{k}$ decreases, and the equilibrium PA becomes uniform.

\section{CONCLUSION}

Using tools of random matrices, we have derived the equilibrium power allocation in a game-theoretic framework applied to asymptotic CDMA with cyclic prefix, under frequency-selective fading. Three receivers are considered: matched filter, MMSE and optimum filter (given by Shannon's capacity). For each user, this power allocation depends only on 


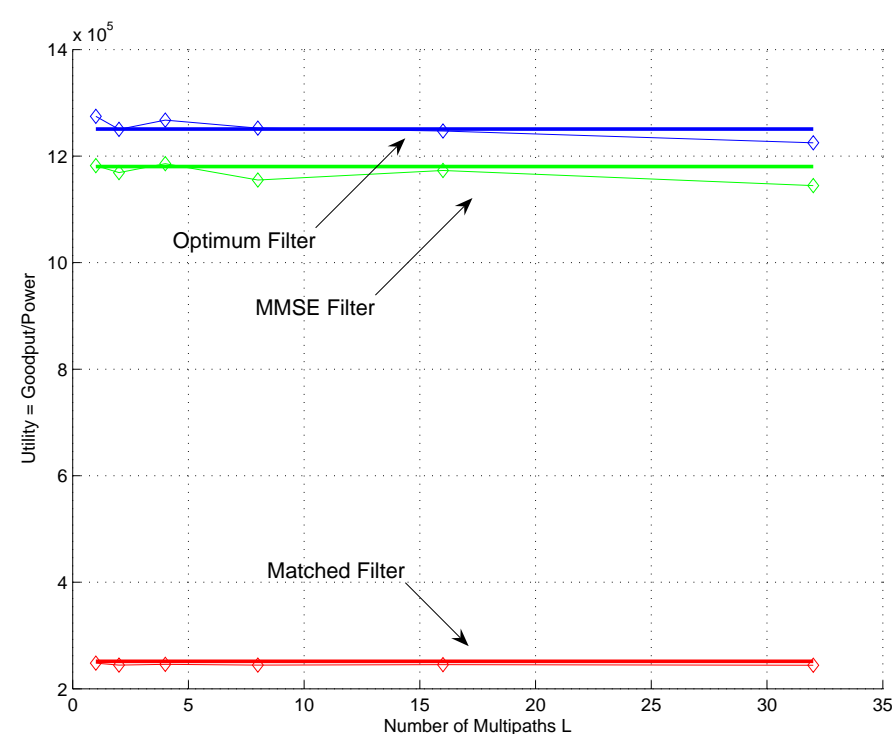

Fig. 1. Comparison of theoretic values and simulations for utilities in the Nash equilibrium.

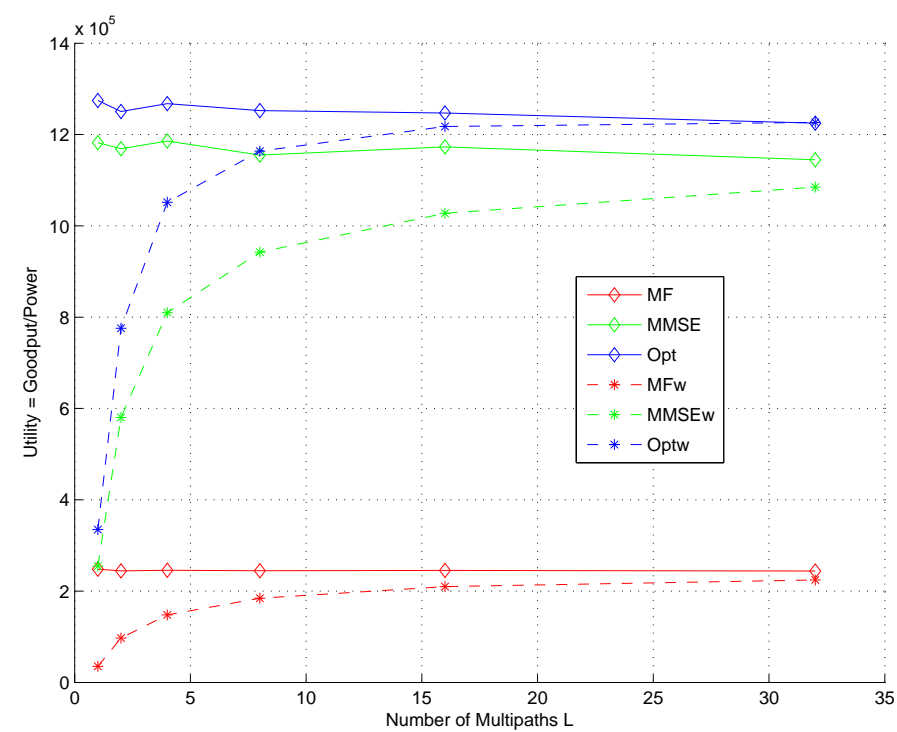

Fig. 2. Simulation of utilities in the Nash equilibrium and constant power allocations versus $L$.

the total energy of the channel of the user under consideration. For a frequency-flat channel, the power allocation among users is dis-uniform, whereas when the number of multipaths increases, the power allocation tends more and more to a uniform one.

\section{APPENDIX}

\section{A. Proof of Prop. 3}

The proof is adapted from [11]. Notice that when $\sigma^{2} \rightarrow \infty$, $C^{\mathrm{OPT}}=0, C^{\mathrm{MMSE}}=0$ and $\beta(x)=0$. Thus we only have to prove that the derivatives of either side of (12) are equal.
Using $\rho(f, x)=P(x)|h(f, x)|^{2}$, (6) can be rewritten

$$
\beta(x)=\int_{0}^{1} \frac{\rho(f, x) d f}{\sigma^{2}+\int_{0}^{\alpha} \frac{\rho(f, y) 2 d y}{1+\beta(y)}} .
$$

From (1), $\int_{0}^{1} \rho(f, x) u\left(f,-\sigma^{2}\right) d f$ satisfies the same implicit equation (33) as $\beta(x)$ and thus

$$
u\left(f,-\sigma^{2}\right)=\frac{1}{\int_{0}^{\alpha} \frac{\rho(f, y) d y}{1+\beta(y)}+\sigma^{2}} .
$$

Using (33) and (34), we can rewrite

$$
\begin{aligned}
\int_{0}^{1} u\left(f,-\sigma^{2}\right) d f-\frac{1}{\sigma^{2}} & =\int_{0}^{1} \frac{1}{\int_{0}^{\alpha} \frac{\rho(f, y) d y}{1+\beta(y)}+\sigma^{2}} d f-\int_{0}^{1} \frac{1}{\sigma^{2}} d f \\
& =\int_{0}^{1} \frac{-\int_{0}^{\alpha} \frac{\rho(f, x)}{1+\beta(x)} d x}{\sigma^{2}\left(\int_{0}^{\alpha} \frac{\rho(f, y) d y}{1+\beta(y)}+\sigma^{2}\right)} d f \\
& =\int_{0}^{\alpha} \frac{-1}{\frac{(1+\beta(x))}{\sigma^{2}}} \int_{0}^{1} \frac{\rho(f, x) d f}{\int_{0}^{\alpha} \frac{\rho(f, y) d y}{1+\beta(y)}+\sigma^{2}} d x \\
& =-\int_{0}^{\alpha} \frac{\beta(x)}{\sigma^{2}(1+\beta(x))} d x .
\end{aligned}
$$

Thus from (10)

$$
\frac{\partial C^{\mathrm{OPT}}}{\partial \sigma^{2}}=-\log _{2}(e) \int_{0}^{\alpha} \frac{\beta(x)}{\sigma^{2}(1+\beta(x))} d x .
$$

Differentiating (7) with respect to $\sigma^{2}$, we obtain

$$
\frac{\partial C^{\mathrm{MMSE}}}{\partial \sigma^{2}}=\log _{2}(e) \int_{0}^{\alpha} \frac{1}{1+\beta(x)} \frac{\partial \beta}{\partial \sigma^{2}}(x) d x .
$$

Let $\pi(x)=\frac{1}{\sigma^{2}(1+\beta(x))}$. From (35) and (36), we obtain

$$
\begin{aligned}
\frac{\partial C^{\mathrm{OPT}}}{\partial \sigma^{2}} & -\frac{\partial C^{\mathrm{MMSE}}}{\partial \sigma^{2}} \\
= & -\log _{2}(e) \int_{0}^{\alpha}\left(\beta(x)+\sigma^{2} \frac{\partial \beta}{\partial \sigma^{2}}(x)\right) \pi(x) d x .
\end{aligned}
$$

From (6), we have

$$
\begin{aligned}
\int_{0}^{\alpha} \sigma^{2} & \beta(x) \frac{\partial \pi}{\partial \sigma^{2}}(x) d x \\
= & \int_{0}^{\alpha} \int_{0}^{1} \frac{\sigma^{2} \rho(f, x) d f}{\sigma^{2}+\int_{0}^{\alpha} \sigma^{2} \rho(f, y) \pi(y) d y} \frac{\partial \pi}{\partial \sigma^{2}}(x) d x \\
= & \int_{0}^{1} \frac{\int_{0}^{\alpha} \rho(f, x) \frac{\partial \pi}{\partial \sigma^{2}}(x) d x}{1+\int_{0}^{\alpha} \rho(f, y) \pi(y) d y} d f \\
= & \frac{1}{\log _{2}(e)} \frac{\partial}{\partial \sigma^{2}} \int_{0}^{1} \log _{2}\left(1+\int_{0}^{\alpha} \rho(f, y) \pi(y) d y\right) d f .
\end{aligned}
$$

Observing that

$$
\begin{aligned}
\int_{0}^{\alpha}\left(\beta(x)+\sigma^{2} \frac{\partial \beta}{\partial \sigma^{2}}(x)\right) \pi(x) & +\sigma^{2} \beta(x) \frac{\partial \pi}{\partial \sigma^{2}}(x) d x \\
= & \frac{\partial}{\partial \sigma^{2}} \int_{0}^{\alpha} \sigma^{2} \beta(x) \pi(x) d x
\end{aligned}
$$

we obtain (12) from Prop. 3. 


\section{B. Proof of Prop. 5}

Given $C^{\star}$, we can use (12) to obtain a Nash equilibrium power allocation in the following way. We rewrite (12) assuming that the target SINR for the MMSE filter is $\beta^{+}$.

$$
\begin{array}{r}
\alpha \log _{2}\left(1+\beta^{+}\right)-\alpha \log _{2}(e) \frac{\beta^{+}}{1+\beta^{+}} \\
+\log _{2}\left(1+\frac{1}{\sigma^{2}\left(1+\beta^{+}\right)} \int_{0}^{\alpha} P(y)|h(y)|^{2} d y\right) \\
=\alpha \log _{2}\left(1+\beta^{\star}\right) .
\end{array}
$$

In the left-hand side of (38), $P(y)$ is given by a MMSE power allocation similar to the one given by (20). Hence, the term $\int_{0}^{\alpha} P(y)|h(y)|^{2} d y$ in (38) does not depend on the actual realizations of the channels. Replacing $\beta^{\star}$ by $\beta^{+}$in (19), we obtain that $\int_{0}^{\alpha} P(y)|h(y)|^{2} d y=\frac{\alpha \sigma^{2} \beta^{+}}{1-\alpha \frac{\beta^{+}}{1+\beta^{+}}}$, which gives us (22). Replacing $\beta^{\star}$ by $\beta^{+}$in (20), we obtain the power allocation (21).

\section{Expectation of the random variable (25)}

For each user $j$, there are $L>1$ paths with respective attenuations $h_{\ell}\left(\frac{j}{N}\right), \ell=1, \ldots, L$, which are i.i.d. complex random variables with mean zero and even distributions of the real and imaginary parts. The Fourier transform of those attenuations is $h_{n j}=h\left(\frac{n}{N}, \frac{j}{N}\right)=\sum_{\ell=1}^{L} h_{\ell}\left(\frac{j}{N}\right) e^{-2 \pi i \frac{n}{N}(\ell-1)}$. The total energy of the paths is $E_{j}=\sum_{\ell=1}^{L}\left|h_{\ell}\left(\frac{j}{N}\right)\right|^{2}$.

We want to show that the expectation of the random variable $\frac{1}{K} \sum_{j=1}^{K} \frac{\left|h_{n j}\right|^{2}}{E_{j}}$ is equal to 1 . By expanding the expression of $h_{n j}$, this is equivalent to showing that the expectation of the random variable

$$
\frac{h_{\ell}\left(\frac{j}{N}\right) h_{\ell^{\prime}}\left(\frac{j^{\prime}}{N}\right)}{E_{j}}
$$

is equal to 0 . Denoting by $p(\cdot)$ the distribution of $h_{\ell}=h_{\ell}\left(\frac{j}{N}\right)$, this expectation is equal to the $L$-dimensional integral of

$$
\frac{h_{\ell} h_{\ell^{\prime}}}{\left|h_{\ell}\right|^{2}+\left|h_{\ell^{\prime}}\right|^{2}+\sum_{k \neq \ell, \ell^{\prime}}\left|h_{k}\right|^{2}} p\left(h_{\ell}\right) p\left(h_{\ell^{\prime}}\right) \prod_{k \neq \ell, \ell^{\prime}} p\left(h_{k}\right)
$$

which is an odd function of $h_{\ell}$. Its integral is therefore 0 , which proves the desired result.

\section{REFERENCES}

[1] D. Tse and S. Hanly, "Multiaccess Fading Channel-Part I: Polymatroid Structure, Optimal Resource Allocation and Throughput Capacity," IEEE Trans. on Information Theory, vol. 44, no. 7, pp. 2796-2815, Nov. 1998.

[2] E. Altman, T. Boulogne, R. E. Azouzi, T. Jimenez, and L. Wynter, "A survey on networking games," Computers and Operations Research, 2005.

[3] L. Lai and H. E. Gamal, "The Water-Filling Game in Fading Multiple Access Channels," submitted to IEEE Transactions on Information Theory, 2005.

[4] F. Meshkati, H. Poor, S. Schwartz, and N. Mandayam, "An EnergyEfficient Approach to Power Control and Receiver Design in Wireless Data Networks," IEEE Trans. on Communications, vol. 53, no. 11, pp. 1885-1894, Nov. 2005.

[5] F. Meshkati, M. Chiang, H. Poor, and S. Schwartz, "A Game-Theoretic Approach to Energy-Efficient Power Control in Multi-Carrier CDMA Systems," IEEE Journal on Selected Areas in Communications, 2006.

[6] V. L. Girko, "Theory of Random Determinants," Kluwer Academic Publishers, Dordrecht, The Netherlands, 1990.

[7] J. Silverstein and Z. Bai, "On the Empirical Distribution of Eigenvalues of a Class of Large Dimensional Random Matrices," J. Multivariate Anal., vol. 54, no. 2, pp. 175-192, 1995.

[8] B. M. Hochwald, T. L. Marzetta, and V. Tarokh, "Multiple-Antenna Channel Hardening and Its Implications for Rate Feedback and Scheduling," IEEE Trans. on Information Theory, vol. 50, no. 9, pp. 1893-1909, Sept. 2004.

[9] A. Tulino and S. Verdú, "Random matrix theory and wireless communications," Foundations and Trends in Communications and Information Theory, vol. 1, no. 1, 2004.

[10] D. Tse and S. Hanly, "Linear Multiuser Receivers: Effective Interference, Effective Bandwidth and User Capacity," IEEE Trans. on Information Theory, vol. 45, no. 2, pp. 641-657, Mar. 1999.

[11] A. Tulino, L. Li, and S. Verdú, "Spectral Efficiency of Multicarrier CDMA," IEEE Trans. on Information Theory, vol. 51, no. 2, pp. 479505, February 2005.

[12] J. Wardrop, "Some Theoretical Aspects of Road Traffic Research Communication Networks," in Proc. Inst. Civ. Eng., vol. 2, no. 1, 1952, pp. 325-378.

[13] A. Haurie and P. Marcotte, "On the Relationship between Nash-Cournot and Wardrop Equilibria," Networks, vol. 15, pp. 295-308, 1985.

[14] R. Gray, "Toeplitz and Circulant Matrices: A Review," Foundations and Trends in Communications and Information Theory, vol. 2, no. 3, pp. 155-239, 2006.

[15] K. Fazel and L. Papke, "On the Performance of Convolutionally-Coded CDMA/OFDM for Mobile Communication System," in Proceedings of IEEE PIMRC, Yokohama, Japan, Sept. 1993, pp. 468-472.

[16] J. Lindner, "MC-CDMA and its Relation to General Multiuser/Multisubchannel Transmission Systems," in International Symposium on Spread Spectrum Techniques \& Applications, Mainz, Germany, Sept. 1996, pp. 115-121.

[17] J. Bingham, "Multicarrier Modulation for Data Transmission: An Idea Whose Time Has Come," IEEE Communications Magazine, vol. 28, no. 5, pp. 5-14, May 1990.

[18] J. Evans and D. Tse, "Large System Performance of Linear Multiuser Receivers in Multipath Fading Channels," IEEE Trans. on Information Theory, pp. 2059-2078, Sept. 2000.

[19] W. Hachem, "Simple Polynomial Detectors for CDMA Downlink Transmissions on Frequency-Selective Channels," IEEE Trans. on Information Theory, vol. 50, no. 1, pp. 164-171, January 2004.

[20] R. Müller, "Multiuser Receivers for Randomly Spread Signals: Fundamental Limits with and without Decision-Feedback," IEEE Trans. on Information Theory, vol. 47, no. 1, pp. 268-283, Jan. 2001.

[21] C. Saraydar, N. B. Mandayam, and D. J. Goodman, "Efficient Power Control via Pricing in Wireless Data Networks," IEEE Trans. on Communications, vol. 50, no. 2, pp. 291-303, Feb. 2002. 